\title{
The Transcutaneous Vagus Nerve Stimulation Output of the NuCalm Device of Solace Lifesciences Is Found to Be a Multifractal and Therefore It Is Indicated in the Treatment of Heart Rate Variability in the Dysfunction of Autonomic Nervous System in Anxiety, Depression and Stress
}

\author{
Sergio Conte1, Fang Wang2, Nicoletta Sala 3 , Francesco Losito Casciaro ${ }^{1,4}$, Franco Orsucci 5 , \\ Giuseppe Serafini6, Ferda Kaleagasioglu',7, Leonardo Mendolicchio, ${ }^{1,8}$, Rich Norman1, \\ Elio Conte ${ }^{1}$ \\ ${ }^{1}$ School of Advanced International Studies on Applied Theoretical and Non Linear Methodologies of Physics, Bari, Italy \\ ${ }^{2}$ College of Science/Agricultural Mathematical Modeling and Data Processing Center, \\ Hunan Agricultural University, Changsha, China \\ ${ }^{3}$ University of Lugano, ISSI/Cerfim, Lugano, Swizerland \\ ${ }^{4}$ Department of Biomedical Sciences and Human Oncology, University of Bari, Bari, Italy \\ ${ }^{5}$ Division of Psychology, University College, London, England \\ ${ }^{6}$ Tor Vergata University, Rome, Italy \\ ${ }^{7}$ Faculty of Medicine, Near East University, North Nicosia, Turkish Republic of Northern Cyprus \\ ${ }^{8}$ Clinic Villa Miralago, ASL Varese, Italy \\ Email: elio.conte@fastwebnet.it
}

How to cite this paper: Conte, S., Wang, F., Sala, N., Casciaro, F.L., Orsucci, F., Serafini, G., Kaleagasioglu, F., Mendolicchio, L., Norman, R. and Conte, E. (2017) The Transcutaneous Vagus Nerve Stimulation Output of the NuCalm Device of Solace Lifesciences Is Found to Be a Multifractal and Therefore It Is Indicated in the Treatment of Heart Rate Variability in the Dysfunction of Autonomic Nervous System in Anxiety, Depression and Stress. Journal of Behavioral and Brain Science, 7, 532-543. https://doi.org/10.4236/jbbs.2017.711038

Received: October 8, 2017

Accepted: November 17, 2017

Published: November 21, 2017

\begin{abstract}
We have investigated the $\mathrm{Nu}$ Calm Transcutaneous Vagus Nerve Stimulation device of the NuCalm Solace Lifesciences founding that it gives a multifractal output. A diminishing fractal/multifractal HRV is consistently reported in literature as related to a serious Autonomic Nervous System (ANS) dysfunction that of course we observe in several psychological and psychiatric disorders. Therefore, we suggest the investigators to apply such a device in subjects affected from anxiety, depression and stress using the method of inducing tVNS stimulation.
\end{abstract}

Keywords

Depression, Anxiety, Stress, Transcutaneous Vagus Nerve Stimulation, 
Copyright () 2017 by authors and Scientific Research Publishing Inc. This work is licensed under the Creative Commons Attribution International License (CC BY 4.0). http://creativecommons.org/licenses/by/4.0/

\section{Neuromodulation, Heart Rate Variablity, Multifractals}

\section{Introduction}

The autonomic nervous system (ANS) affects every physiologic system; it has a highly complex anatomy, control, and physiological influence. Its complex dynamics is fundamental in responses to a wide range of physical, emotional and psychological stressors. Impairment of autonomic function is presented in a variety of clinical psychological and psychiatric disorders that can be highly debilitating and at risk for subjects. Accurate studies are required for advanced understanding of this neural system in its diversity and in its impact on physiologic, psycho-physiologic and clinical outcomes [1].

Heart Rate Variability (HRV) is used to quantify activity of the autonomic nervous system since cardiac rhythm is modulated by the two modulating components of the autonomic nervous system (ANS), the sympathetic and parasympathetic systems, which exert antagonistic effects. Sympathetic dominance occurs during stressful conditions and psychological and psychiatric disorders, whereas the vagal modulation induces normal heart rate dynamics and the correct its intrinsic variability. Accordingly, heart rate and RR interval variability analysis (HRV) enable the study of autonomic influence as well as the effects of a number of psychological and psychiatric disorders and stress level on sympathetic and vagal outputs (HRV analysis). From a clinical point of view, the treatment of such mentioned psychological disorders requires often the continued use of drugs and quite often coupled to psychotherapy. The use of alternative clinical approach is of importance especially in the several cases in which patients evidence conditions having pharmacologically resistance [2].

Traditionally, the idea that we can influence neuronal activity with electrical pulses is not new. Earlier this century, patients were treated, and still are, with electroconvulsive therapy as a treatment for severe depression [3].

Presently, electrical stimulation of the vagus nerve (VNS) is an FDA-approved therapy tool for both refractory depression and epilepsy [4] [5] [6]. It has been used, as well, for a number of psychological and psychiatric disorders. As supported by neuroimaging studies, VNS broadly affects different parts of the brain, including the thalamus, cerebellum, orbito-frontal cortex, limbic system, hypothalamus, and medulla [7] [8] [9] [10]. Transcutaneous vagus nerve stimulation (tVNS) was initially arranged by implanting a neurostimulating device connected to an electrode located along the cervical branch of the vagus nerve. In principle, this procedure may have negative aspects, such as coughing during stimulation, hoarseness, general operational, and anesthesiological risks, and high costs. In order to minimize adverse effects, a new non-invasive neurostimulating procedure, called transcutaneous vagus nerve stimulation (tVNS) stimulation, has been developed. 
Consequently, it becomes of fundamental importance to analyze in detail such innovating procedure under the technical as well as clinical profiles. In our studies we used the NuCalm tVNS device (http://www.nucalm.com/). This device applies two gel electrode patches to the neck below the ear lobe (right and left) for transcutaneous stimulation of the afferent auricular branch of the vagus nerve (ABVN). The device received FDA approval, satisfying essential health and safety requirements. Transcutaneous vagus nerve stimulation (tVNS) targets the cutaneous receptive field of the ABVN at the outer ear [2]. Ear lobe vagal stimulation does not create adverse effects [11], but it does induce modulating effects on heart rate, blood pressure, or peripheral microcirculation during the stimulation procedure. The use of applied electrodes near both right and left ear lobes assures afferent and efferent induction since the right vagus nerve has efferent fibers to the heart. Of course, the use of the device is not recommended in the case of pregnancy, and is contraindicated for persons with metal in the body, such as pacemaker and ventricular assist devices or brain and cochlear implants. Actually, the NuCalm system (http://www.nucalm.com/) results in a combined system of components that work together to bring the body to a deep state of relaxation. First the subject applies a NuCalm topical cream that has been designed to induce relaxes by counteracting adrenaline. The proprietary formula is only available in the NuCalm system and includes structured, nutrient-sourced building blocks that rapidly create a natural relaxation response. The treatment continues by applying tVNS and noise-dampening headphones with binaural beats used to bring brain to synchronize at proper frequency bands in the interval $(12 \mathrm{~Hz}-4 \mathrm{~Hz})$. The whole treatment uses light-blocking eye masks with the obvious meaning to reduce perceptive effects enhancing alpha and related relaxing bands. Since it is intuitive that the use of a such combined procedure may produce only positive treatment effects, there is to agree on the evident benefit to use the mentioned cream as well as to add brain entrainment synchronizing in the frequency bands $(4-12 \mathrm{~Hz})$, it remains the basic interest to study the tVNS device from one hand under its technical profile and when applied to subjects having the above mentioned psychological disorders. Recently, we performed our studies by using the tVNS NuCalm device on subjects affected from anxiety or depressions [12]. The study was performed on subjects having a serious ANS dysfunction as evidenced by our HRV analysis and examining the effect on the ANS of the NuCalm treatment for thirty minutes. During and after the tVNS treatment, the considered subjects evidenced a net improvement in ANS as evidenced by HRV analysis. This encouraging result has directed our subsequent research under different directions. In fact, it is evident that in order to accreditate such treatment that, as said, has resulted very promising, we have to analyze in detail the basic features of such tVNS device, inspecting not only its technical features but analizing in detail the features of its output signal, attempting to delineate its basic features and the reasons because from a physiological as well as clinic profiles, such device induces the expected benefits. This is done in the tentative to introduce a solid scientific base of reliability, first of all in relation to 
the device that may be used in such so delicate treatments and obviously in relation to an explanation of the reasons because, when applied, such device induces such observed positive effects. The purpose of the present paper is to expose the results that we have obtained on the first step, which is the investigation of the basic technical features of the NuCalm tVNS device arriving to delineate what are the physiological and clinical reasons because it runs suitably. The investigation leads to the conclusion that the tVNS NuCalm device has a multifractal output signal and its multifractal output structure may provide an improvement in subjects with anxiety, depression or stress.

\section{Materials and Methods}

The first step was to evaluate the technical features of the instrument. It furnishes in output different currents at the electrodes and the minimum value of 0.1 $\mathrm{mA}$ was selected by us for our investigations. In Figure 1 we report the results of our oscilloscope monitoring that was performed for about twenty four hours of device running. The Figure 1 evidences the oscilloscope monitoring of the output $\mathrm{NuCalm}$ device that is evidenced in the foreground with the two electrodes ready to be used.

The NuCalm device output was also constantly monitored by a Welleman PCLAB 2000 SE computer interface with recording of the output values.

In Figure 2 we have the complete output signal given from the device.

The instrument is given in Figure 3 while in Figure 4 it is illustrated the manner to use it.

The current of the instrument varies in the range of $0.1-1.5 \mathrm{~mA}$ and a stimulation current of $0.1 \mathrm{~mA}$ was selected in the case of the present study.

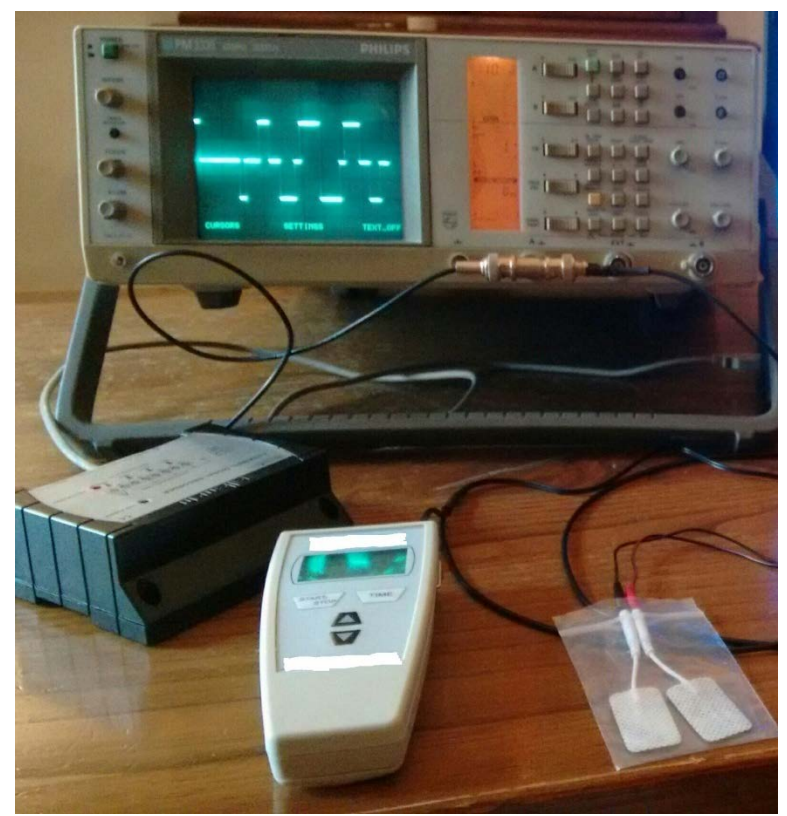

Figure 1. Arrangement of the NuCalm Device monitored with an oscilloscope for twenty four hours. 


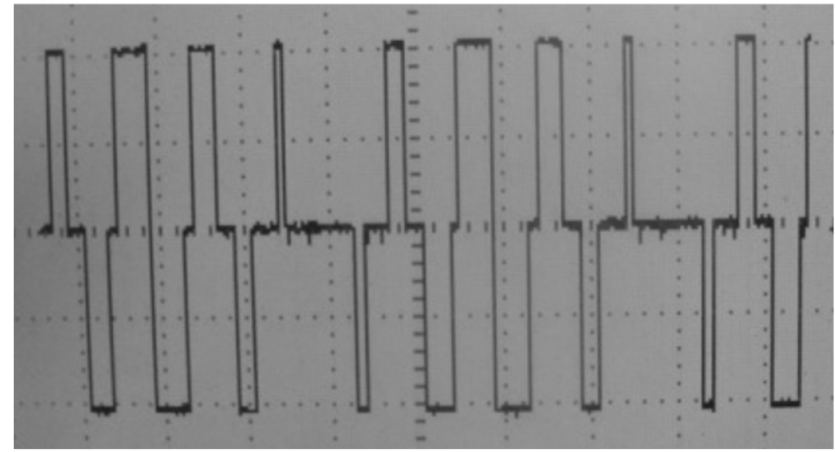

Figure 2. Time behavior of the complete output signal of the device.

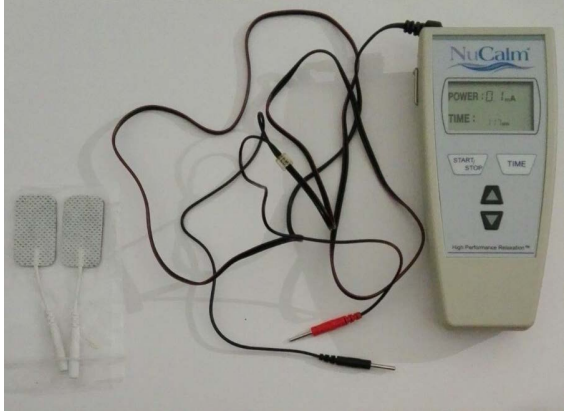

(a)

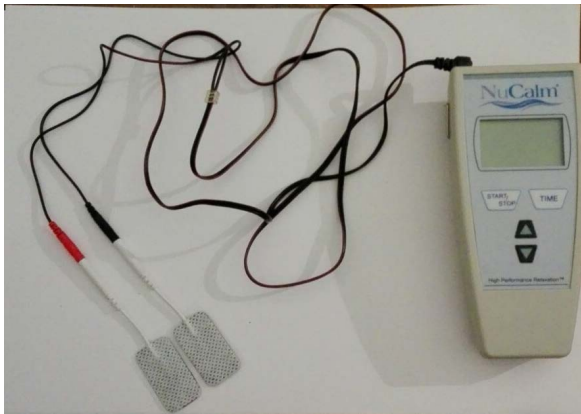

(b)

Figure 3. (a) The NuCalm device; (b) The NuCalm with the electrodes ready to be used.

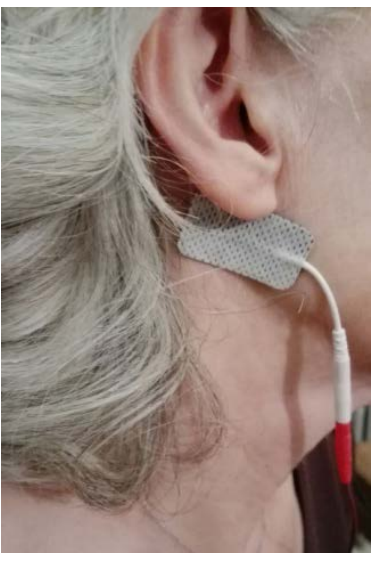

(a)

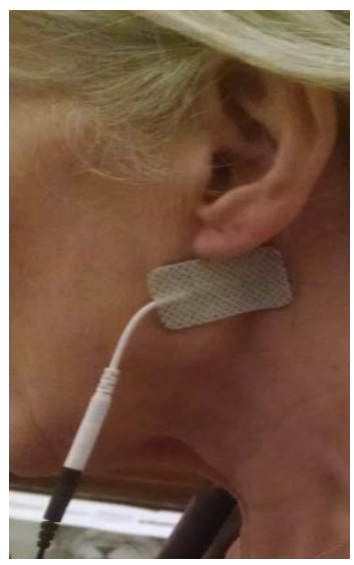

(b)

Figure 4. (a) The NuCalm device with connected red (right) electrode; (b) The NuCalm device with connected the black (left) electrode.

The recorded time series data were analyzed by using the method of the Multi Fractal Detrended Fluctuation Analysis (MF-DFA-1D) of Kantelhardt et al. 2002 [13] see in detail [12] [14] [15] [16] [17]

\section{Results}

The first step was to ascertain the stability of the output. The system resulted 
stable. We fixed a reasonable threshold value $M$ and, indicating the output signal by $x(t)$, always we found

$$
\left|x(t)-x\left(t+T_{i}\right)\right| \leq M .
$$

with $T_{i}$ being the periods of the signal.

The next step was to analyze the obtained output.

We give here the obtained results.

The first estimated variable was the Hurst exponent as detailed in (1).

The results are given in Figure 5.

$h(q)$ shows monotonically decreasing values as function of $q$. The estimated Hurst exponents is $H=1.9158$.

Soon after we examined the function $\tau \equiv \tau(q)$ as detailed in (2) (Figure 6).

$\tau(q)$ evidences its typical non linear dependence upon $q$.

Let us examine now $\alpha \equiv \alpha(q)$ as detailed in (3). The results are given in Figure 7.

Finally, we examined the spectrum, $f(\alpha)$, as detailed in (4). The result is given in Figure 8.

The results given from Figure 5 to Figure 8 indicate only one result.

The output signal of the NuCalm tVNS device is a multifractal. This output signal gives the following multifractal strength, $\Delta \alpha=\alpha_{\max }-\alpha_{\min }=0.5845$ and $\Delta f=f\left(\alpha_{\max }\right)-f\left(\alpha_{\min }\right)=0.1931$.

\section{Discussion}

To have discovered that the NuCalm tVNS has been able to realize a device that gives an output multifractal time dynamics, represents a remarkable note of merit for this instrument. Let us attempt to explain the reasons in detail.

The fractals were first introduced by Benoît Mandelbrot in 1975 [18] [19] and fractal geometry characterizes systems having basic irregular and self-similarity at all scales. This is to say that a fractal structure has the property that a small portion of the system evidences the same complexity as the entire system. In Nature we find fractals and multifractals. Fractal is a system whose scaling

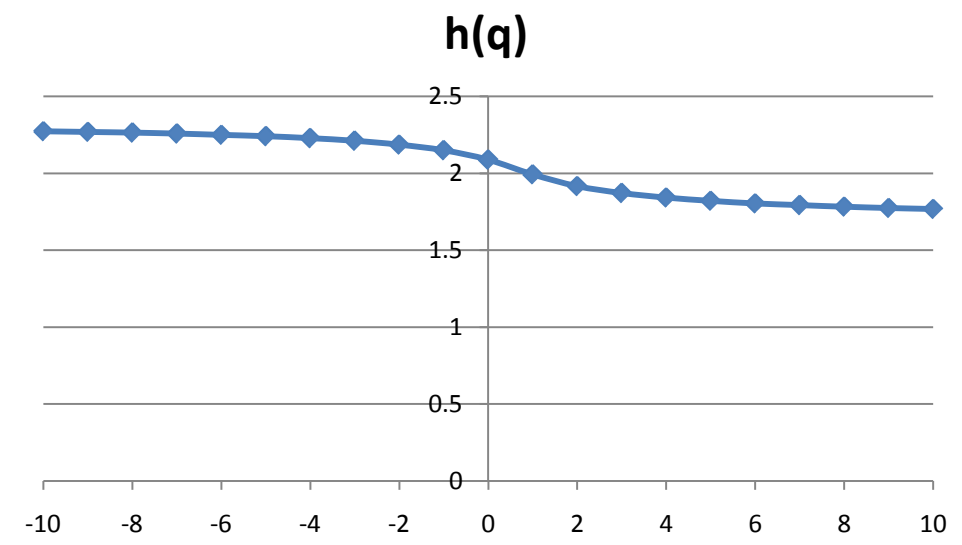

Figure 5. Estimated Hurst exponents. 


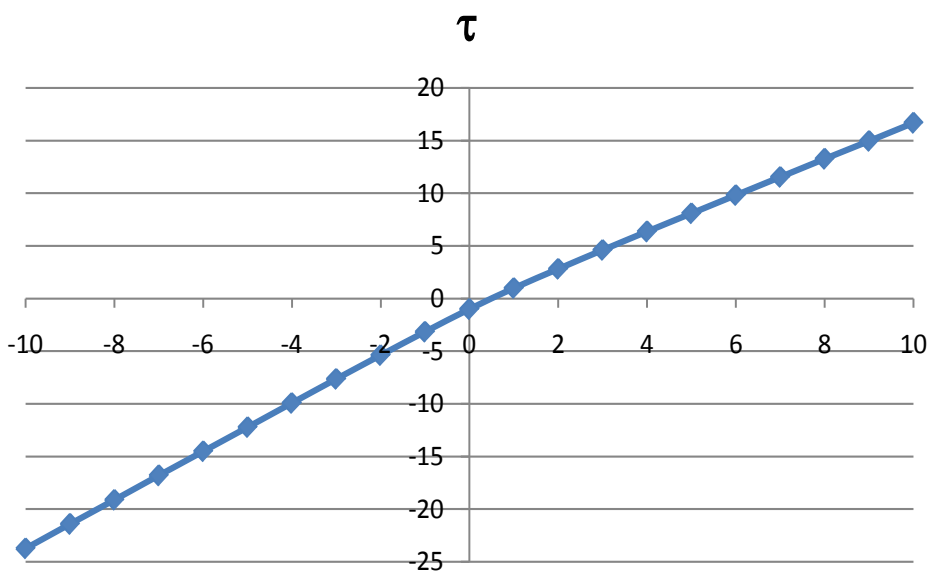

Figure 6. Estimated $\tau(q)$ function.

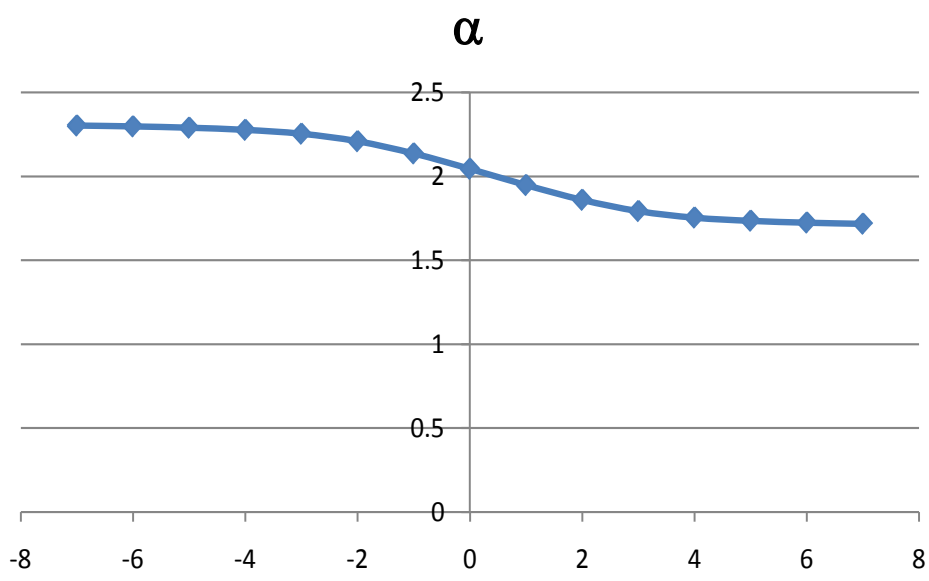

Figure 7. The estimated function $\alpha(q)$.

$f(\alpha)$

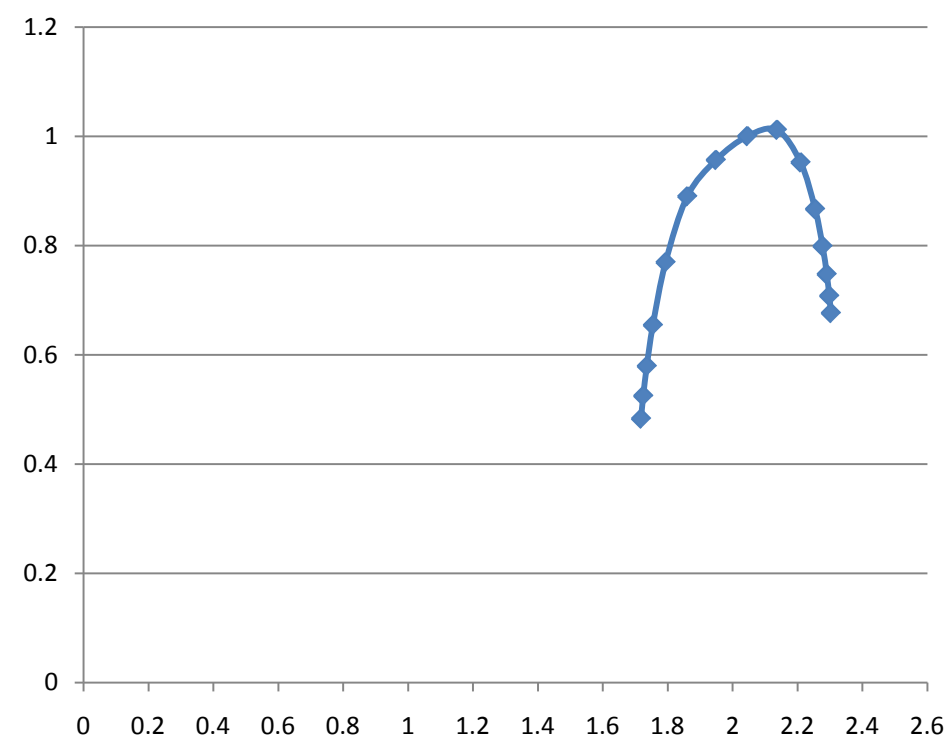

Figure 8. The multifractal spectrum, $f(\alpha)$. 
properties are the same in different scales. Multifractals are very complex self-similar systems since they are weighted fractals that have different non-integer dimensions. Consequently a multifractal system is a generalization of a fractal system. In this case a single scaling exponent is not sufficient to describe its structure; really, a continuous spectrum of exponents (the so called singularity spectrum) is needed [20]. The salient feature that relates the finality of our paper is that in recent years multifractals have been discovered in space as well as time domains, and the multifractal analysis has been widely applied to diverse fields of biology and medicine as the analysis of DNA sequences, neuron spiking, human gait. Fractal properties have been found also in the human neuromuscular system, they have been observed in ECG, electroencephalogram (EEG) recordings of brain waves as well as in recordings of human movement, and eye movements [21]-[28]. The central point is that multifractals have been found also in heart rate dynamics and HRV that are actually the basic objects of the present paper [1] [29]-[35]. The arising conclusion is that multifractal structures represent a property of the system dynamics of biological and medical systems. The clinic criterium is that the pathology usually modifies or can disrupt this fractal or multifractal dynamics resulting in the alteration of its fractal properties. The MFDFA method that we have used, was first conceived by Kantelhardt et al. 2002 [13] and, as previously seen, it enables to estimate if multifractal behavior exists in the fluctuation function

$$
F_{q}(s) \propto s^{h(q)}
$$

being $q$ the order of the fluctuation.

In general the exponent $h(q)$ depends on $q$. For stationary time series $h(2)$ is identical with the Hurst exponent H. $h(q)$ is said to be the generalized Hurst exponent. A mono fractal time series is characterized by unique $h(q)$ for all values of $q$. In the multifractal case we obtain instead a distribution of values as in Figure 3.

The generalized Hurst exponent $h(q)$ is related to the classic scaling exponent $\tau(q)$ by the relation

$$
\tau(q)=q h(q)-1
$$

A monofractal series with long range correlation is characterized by linearly dependent $q$ order exponent $\tau(q)$ with a single Hurst exponent. On the contrary, multifractal signal has multiple Hurst exponent and $\tau(q)$ depends non-linearly on $q$ as we obtain in Figure 4.

The singularity spectrum $f \alpha$ is related to $h(q)$ by

$$
\alpha=h(q)+\alpha h^{\prime}(q)
$$

and we obtain it in Figure 5 and

$$
f(\alpha)=[\alpha-h(q)]+1
$$

and we obtain it in Figure 6.

In conclusion, we have given demonstration that the output signal of the 
tVNS Nu Calm device produces a multifractal. We have to evidence the reason because this result is so important.

We have previously said that the heart rate regulation in healthy humans is known to exhibit complex variability over an extensive time dynamic range. Specifically, in the beat-to-beat RR interval, the variability of such heart rhythm is intrinsic and of fundamental importance. In previous papers we have also shown that it exhibits scale-free characteristics [36] [37] [38] and Ivanonv et al. and Sassi et al. have shown that it is of multifractal type [39] [40]. The conclusion is that scale-free component of the heart rate variability (HRV) is of basic importance. It also develops relevant information in the clinical context since a diminishing fractal HRV is consistently reported as related to a serious ANS dysfunction that of course we observe in several psychological disorders. This is to say that different factors influence HRV scale-free dynamics, and in such context, the potential contribution of the central nervous system (CNS) cannot be ignored. On the other hand the sympathetic (SNS) and parasympathetic (PNS) branches of the autonomic nervous system (ANS) realize a complex interaction between SNS and PNS that requires advanced investigation. In fact, it has been studied that SNS blockade by drugs has a minor effect on the multifractal property of HRV. Therefore we have arrived to the central point: stimulation of the vagus nerve by a signal that is intrinsically multifractal induces a strong heart related neuro-modulation that enables the subject to recover ANS dysfunction related to his/her psychological disorder. In this framework, the use of tVNS based on a Nu Calm mutifractal output signal results is of decisive importance. We stimulate researchers to experimentally verify such new line of research by using the tVNS NuCal device that, as previously observed, has a valuable output multifractal signal able to induce the expected neuro-modulation in subjects having anxiety, depression and stress. Experimental applications will be able to evaluate also the limitations in the different clinical cases of application.

\section{References}

[1] Bunde, A., Havlin, S., Kantelhardt, J.W., Penzel,T., Peter, J.H. and Voigt, K. (2000) Corrrelated and Uncorrelated Regions in Heart-Rate Fluctuations during Sleep. Physical Review Letters, 85, 3736-3739. https://doi.org/10.1103/PhysRevLett.85.3736

[2] Ellrich, J. (2011) Transcutaneous Vagus Nerve Stimulation European Neurological Review, 6, 262-264. https://doi.org/10.17925/ENR.2011.06.04.254

[3] Fink, M. (1984) The Origins of Convulsive Therapy. American Journal of Psychiatry, 141, 1034-1041. https://doi.org/10.1176/ajp.141.9.1034

[4] Schachter, S.C. and Saper, C.B. (1998) Vagus Nerve Stimulation. Epilepsia, 39, 677-686. https://doi.org/10.1111/j.1528-1157.1998.tb01151.x

[5] Ben-Menachem, E. (2002) Vagus-Nerve Stimulation for the Treatment of Epilepsy. The Lancet Neurology, 1, 477-482. https://doi.org/10.1016/S1474-4422(02)00220-X

[6] Grimm, S. and Bajbouj, M. (2010) Efficacy of Vagus Nerve Stimulation in the Treatment of Depression. Expert Review of Neurotherapeutics, 10, 87-92.

https://doi.org/10.1586/ern.09.138 
[7] Chae, J.H., Nahas, Z., Lomarev, M., Denslow, S., Lorberbaum, J.P., Bohning, D.E. and George, M.S. (2003) A Review of Functional Neuroimaging Studies of Vagus Nerve Stimulation (VNS). Journal of Psychiatric Research, 37, 443-455. https://doi.org/10.1016/S0022-3956(03)00074-8

[8] Pardo, J.V., Sheikh, S.A., Schwindt, G.C., Lee, J.T., Kuskowski, M.A., Surerus, C., Lewis, S.M., Abuzzahab, F.S., Adson, D.E. and Rittberg, B.R. (2008) Chronic Vagus Nerve Stimulation for Treatment Resistant Depression Decreases Resting Ventromedial Prefrontal Glucose Metabolism. Neuroimage, 42, 879-889.

https://doi.org/10.1016/j.neuroimage.2008.04.267

[9] Vonck, K., De Herdt, V., Bosman, T., Dedeurwaerdere, S., Van Laere, K. and Boon, P. (2008) Thalamic and Limbic Involvement in the Mechanism of Action of Vagus Nerve Stimulation, a SPECT Study. Seizure, 17, 699-706. https://doi.org/10.1016/j.seizure.2008.05.001

[10] Kosel, M., Brockmann, H., Frick, C., Zobel, A. and Schlaepfer, T.E. (2011) Chronic Vagus Nerve Stimulation for Treatment-Resistant Depression Increases Regional Cerebral Blood flow in the Dorsolateral Prefrontal Cortex. Psychiatry Research, 191, 153-159. https://doi.org/10.1016/j.pscychresns.2010.11.004

[11] Kraus, T., Hosl, K., Kiess, O., Schanze, A., Kornhuber, J. and Forster, C. (2007) Bold fMRI Deactivation of Limbic and Temporal Brain Structures and Mood Enhancing Effect by Transcutaneous Vagus Nerve Stimulation. Journal of Neural Transmission, 114, 1485-1493. https://doi.org/10.1007/s00702-007-0755-Z

[12] Conte, S., Wang, F., Altamura, M., Giuliani, A., Bellomo, A., Serafini, G., Orsucci, F., Kaleagasioglu, F., Mendolicchio, L., Losito Casciaro, F., Losito, A.M., Norman, R. and Conte, E. (2017) An Analysis of Heart Rate variability (HRV) in Frequency Domain during the Treatment by Transcutaneous Vagus Nerve Stimulation (tVNS), in Progress on Physiological Measurements.

[13] Kantelhardt, J.W., Zschiegner, S.A., Bunde, E.K., Havlin, S., Bunde, A. and Stanley, H.E. (2002) Multifractal Detrended Fluctuation Analysis of Non Stationary Time Series. Physica A, 316, 87-114.

[14] Vena, A., Conte, E., Perchiazzi, G., Federici, A. and Zbilut, J.P. (2004) Detection of Physiological Singularities in Respiratory Dynamics Analyzed by Recurrence Quantification Analysis of Tracheal Sounds. Chaos, Solitons \& Fractals, 22, 869-881.

[15] Conte, E., Federici, A. and Zbilut, J.P. (2009) A New Method Based on Fractal Variance Function for Analysis and Quantification of Sympathetic and Vagal Activity in Variability of $R-R$ Time Series in ECG Signals. Chaos, Solitons \& Fractals, 41, 1416-1426.

[16] Conte, E., Federici, A. and Zbilut, J.P. (2004) On a Simple Case of Possible Non-Deterministic Chaotic Behavior in Compartment Theory of Biological Observables. Chaos, Solitons \& Fractals, 22, 277-284.

[17] Casciaro, F., Laterza, V., Conte, S., Pieralice, M., Federici, A., Todarello, O., Orsucci, F. and Conte, E. (2013) Alpha-Rhythm Stimulation using Brain Entrainment Enhances Heart Rate Variability in Subjects with Reduced HRV. World Journal of Neuroscience, 3, 213-220. https://doi.org/10.4236/wjns.2013.34028

[18] Mandelbrot, B.B. (1975) Les Objects Fractals: forme, hasard et dimension [Fractal Objects: Form, Chance and Dimension]. Flammarion, Paris.

[19] Mandelbrot, B.B. (1982) Fractal Geometry of Nature. Freeman, New York.

[20] Hausdorff, J.M., Ashkenazy, Y., Peng, C.K., Ivanov, P.C., Stanley, H.E. and Goldberger, A.L. (2001) When Human Walking Becomes Random Walking: Fractal Analysis and Modeling of Gait Rhythm Fluctuations. Physica A, 302, 138-147. 
[21] Goldberger, A.L., Peng, C.K. and Lipsitz, L.A. (2002) What Is Physiological Complexity and How Does It Change with Aging and Disease? Neurobiology of Aging, 23, 23-26.

[22] Scafetta, N., Griffin, L. and West, B.J. (2003) Hölder Exponent Spectra for Human Gait. Physica A, 328, 561-583.

[23] Scafetta, N., Moon, R.E. and West, B.J. (2007) Fractal Response of Physiological Signals to Stress Conditions, Environmental Changes and Neurodegenerative Diseases. Complexity, 12, 12-17.

[24] Scafetta, N., Marchi, D. and West, B.J. (2009) Understanding the Complexity of Human Gait Dynamics. Chaos, 19, Article ID: 026108. https://doi.org/10.1063/1.3143035

[25] Zhou, P., Li, F., Liu, W.Y. and Yang, M. (2007) Fractal Analysis in Normal EEG and Epileptic EEG of Rats. World Congress on Medical Physics and Biomedical Engineering, 14, 1266-1269. https://doi.org/10.1007/978-3-540-36841-0_307

[26] Van Orden, G.C., Kloos, H. and Wallot, S. (2009) Living in the Pink: Intentionality, Well Being, and Complexity. In: Hooker, C.A., Ed., Handbook of the Philosophy of Science 10: Philosophy of Complex Systems, Elsevier, Amsterdam.

[27] Dutta, S. (2010) EEG Pattern of Normal and Epileptic Rats: Monofractal or Multifractal? Fractals, 18, 425-431. https://doi.org/10.1142/S0218348X10005081

[28] Coey, C.A., Wallot, S., Richardson, M.J. and Van Orden, G. (2012) On the Structure of Measurement Noise in Eye-Tracking. Journal of Eye Movement Research, 5, 1-10.

[29] Ossadnik, S.M., Buldyrev, S.V., Goldberger, A.L., Havlin, S., Mantegna, R.N., Peng, C.K., et al. (1994) Correlation Approach to Identify Coding Regions in DNA Sequences. Biophysical Journal, 67, 64-70.

[30] Peng, C.K., Buldyrev, S.V., Havlin, S., Simons, M., Stanley, H.E. and Goldberger, A.L. (1994) Mosaic Organization of DNA Nucleotides. Physical Review E, 49, 1685-1689. https://doi.org/10.1103/PhysRevE.49.1685

[31] Buldyrev, S.V., Dokholyan, N.V., Goldberger, A.L., Havlin, S., Peng, C.K., Stanley, H.E., et al. (1998) Analysis of DNA Sequences using Methods of Statistical Physics. Physica A, 249, 430-438.

[32] Blesic, S., Milosevic, S., Stratimirovic, D. and Ljubisavljevic, M. (1999) Detrended Fluctuation Analysis of Time Series of a Firing Fusing Motorneuron. Physica $A$, 268, 275-282.

[33] Liu, Z., et al. (1999) The Three-Dimensional Structure of the HRDC Domain and Implications for the Werner and Bloom Syndrome Proteins. Structure, 7, 1557-1566.

[34] Talkner, P. and Weber, R.O. (2000) Power Spectrum and Detrended Fluctuation Analysis: Application to Daily Temperatures. Physical Review E, 62, 150-160. https://doi.org/10.1103/PhysRevE.62.150

[35] Ashkenazy, Y., Baker, D.R., Gildor, H. and Havlin, S. (2003) Nonlinearity and Multifractality of Climate Change in the Past 420000 Years. Geophysical Research Letters, 30, 2146-2149.

[36] Conte, E., Giroldini, W., Laterza, V., Conte, S., Pieralice, M., Casciaro, F., De Masi De Luca, G., Giuliano, A.F.M., Mendolicchio, L. and Todarello, O. (2014) Experimental Results on a New Method for Analysis of Heart Rate Variability. World Journal of Cardiovascular Diseases, 4, 385-389.

https://doi.org/10.4236/wjcd.2014.48048 
[37] Conte, E., Ware, K., Marvulli, R., Ianieri, G., Megna, M, Conte, S., Mendolicchio, L. and Pierangeli, E. (2015) Chaos, Fractal and Recurrence Quantification Analysis of Surface Electromyography in Muscular Dystrophy. World Journal of Neuroscience, 5, 205-257. https://doi.org/10.4236/wjns.2015.54022

[38] Conte, E., Ware, K., Marvulli, R., Ianieri, G., Megna, M., Conte, S., Mendolicchio, L. and Pierangeli, E. (2015a) Heart Rate Variability: On the Importance to Perform HRV Analysis in Subjects Affected from Muscular Dystrophy. World Journal of Cardiovascular Diseases, 5, 141-149. https://doi.org/10.4236/wjcd.2015.56017

[39] Ivanov, P.C.H., Amaral, L.A.N., Goldberger, A.L., Havlin, S., Rosenblum, M.G., Struzik, Z.R. and Stanley, H.E. (1999) Multifractality in Human Heart Beat Dynamics. Nature, 399, 461-465. https://doi.org/10.1038/20924

[40] Sassi, R., Signorini, M.G. and Cerutti, S. (2009) Multifractality and Heart Rate Variability. Chaos, 19, Article ID: 028507. https://doi.org/10.1063/1.3152223 